\title{
« Se faire prendre et se faire pendre »: auteurs du coup, la peine de mort
}

\author{
Thomas DuTOIT
}

Université de Lille

escrituraeimagen@filos.ucm.es

\section{Resumen}

El objetivo de este artículo es leer The Executioner's Song de Norman Mailer a la luz de la justificación kantiana de la pena de muerte. La deconstrucción (derridiana) de la equivalencia entre filosofía y pena de muerte mantenida por Kant sostiene esta lectura de Mailer y de Kant. La rigurosa distincion kantiana entre el adentro y el afuera, ejemplificada en la oposición poena naturalis y poena forensis, se somete al movimiento de la différance, cuyo resultado es que las distinciones que aseguran la autopunición y la heteropunición, criminal y juez, no se sostienen. Ese libro es una lectura, o una re-escritura, deconstructiva de «Del derecho de castigar y de perdonar» de Kant.

Palabras clave: deconstrucción, pena de muerte, Kant, castigar.

\begin{abstract}
The purpose of this paper is read Norman Mailer's The Executioner's Song, in light of Kant's defense of the death penalty. Derrida's deconstruction of Kant's equation of philosophy and the death penalty subtends this reading of Mailer and Kant. Kant's rigorous distinction of inside and outside, as exemplified by his opposition between poena naturalis and poena forensis, submits to the movement of différance, whereby re-assuring distinctions of auto-punishment and hetero-punishment, criminal and judge, do not hold. The Executioner's Song is a deconstructive reading, or re-writing, of Kant's « On the Right to Punish and Grant Clemency ».
\end{abstract}

Key words: Deconstruction, death penalty, Kant, punish. 
Mon titre comporte une erreur, qui est ma faute. Au lieu de « Se faire prendre et se faire pendre », j'aurais dû donner " Prendre et pendre». Car c'est une citation, mais une citation d'un enregistrement, et non pas d'un texte. La citation « Prendre et pendre » vient de l'enregistrement de la première séance du séminaire sur la peine de mort que Jacques Derrida donne entre 1999 et 2001. ${ }^{1}$ Quand il a fallu donner à Cristina de Peretti un titre en vue du colloque, je me suis souvenu d'une phrase que Jacques Derrida prononce lors de la séance, phrase qu'il n'a pas écrite. Ma mémoire était cependant défaillante, mais le lapsus dit déjà tout de ce qui sera mon propos aujourd'hui. Voici le morceau en question de l'enregistrement, dans la colonne droite, et le texte du cours dans la colonne gauche :

\begin{tabular}{|l|l|}
\hline ce que Derrida écrit & Ce que Derrida dit \\
Car s'il en était ainsi, si personne ne songeait & Car s'il en était ainsi, si personne ne songeait \\
à se faire pendre ou à risquer de se faire pen- & d'aucune façon à se faire pendre ou à risquer \\
dre, eh bien il n’y aurait jamais d'assassinat & de se faire pendre, eh bien il n’y aurait jamais \\
ni de peine de mort. Il est vrai que Rousseau & d'assassinat ni de peine de mort. Donc il \\
est plus minutieux dans son expression, car il & n'est pas à exclure qu'il y a des contractants \\
dit « présumer » que personne « ne prémédi- & qui n'excluent pas que c'est leur désir de se \\
te de se faire pendre. » (06.12.1999) & faire pendre. Il est vrai que Rousseau est \\
encore plus minutieux dans son expression, \\
car il dit «présumer » que personne « ne pré- \\
médite de se faire pendre. » Il est possible, il \\
présume, donc c'est une hypothèse, il est \\
possible que des gens veuillent inconsciem- \\
ment se faire pendre, c'est ça ... criminel ... ça \\
veut dire ... prendre et pendre. Mais ce que \\
Rousseau exclut, c'est qu'ils le préméditent, \\
c'est-à-dire que consciemment, ils le calcu- \\
lent d'avance, etc.
\end{tabular}

Prendre (voler, tuer) et pendre, cela revient presque au même. Dans l'instant où Derrida dit «prendre et pendre », il semble apercevoir que le « se faire pendre » de Rousseau pourrait se confondre avec un « se faire prendre », comme si le criminel en commettant un crime aurait voulu être pris, arrêté, attrapé, pour ensuite se faire pendre. C'est cela le sens de la première partie de mon titre : se faire pendre présupposerait un «se faire prendre », se faire prendre en flagrant délit.

Dans la deuxième partie du titre, par « auteur de coups », je voulais indiquer que

\footnotetext{
1 Jacques Derrida, Peine de mort, séminaire donné à l'École des Hautes Études en Sciences Sociales, en 1999-2000 et 2000-2001, sera publié, pour le premier tome, en janvier 2011 (Éditions Galilée), sous la responsabilité de Geoffrey Bennington, Marc Crépon et Thomas Dutoit, et pour le deuxième tome, à l'automne 2011. Toute citation ici du séminaire vient du texte tel qu'il est établi pour version publiée, avec la date de la séance entre parenthèses.
} 
j'allais traiter, lire, commenter d'autres auteurs que Jacques Derrida ou que ceux commentés par Jacques Derrida lui-même dans son cours. " Auteurs de coups » voulait indiquer, Norman Mailer, Ernest Gaines, Harper Lee, Mikal Gilmore, Heinrich von Kleist, auteurs à partir desquels j'écris un livre consacré à la peine de mort, dans le sillage du séminaire de Jacques Derrida. Le présent article se limite à Norman Mailer et à Mikal Gilmore. Pour cela, il sera nécessaire de commenter l'auteur qui d'un point de vue philosophique importe le plus à Derrida, à savoir Immanuel Kant, et y juxtaposer l'auteur qui d'un point de vue psychanalytique occupe une place stratégique, Theodore Reik.

Mais sous ce titre, il y a aussi la citation suivante de Jacques Derrida que j'avais lue mais oubliée :

Le Père est à la fois et il n'est ni Dieu-Homme-Bête. La triple question des perversions criminelles (viol, homosexualité, bestialité) n'est pas loin. Le Père est le Vivant en général (Dieu-Homme-Bête) mais ce Vivant en général, dès lors qu'il est au-dessus de la distinction Vie/Mort est voué à la mort, à être tué par cela même à quoi, à qui il donne naissance. [...] Tout cela, si j'ose dire, du seul et même coup. Une fois pour toutes. Un seul et même coup. Car tout ce séminaire sur la peine de mort pourrait revenir à une seule question, d'un seul et même coup : qu'est-ce qu'un coup, qu'est-ce qu'un seul et même coup? Un seul et même coup porté? La mise à mort, l'exécution comme le meurtre sont chaque fois un coup. Et comme la question du coup rejoint la matrice des questions que nous avons posées la dernière fois au sujet de l'intérêt en général et de ce qui vaut ou ne vaut pas la peine d'être vécu, la question du coup (c.o.u.p.) est aussi la question du prix, du coût (c. o. û. t.), de la peine que ça coûte, comme la question du coup qui coupe, comme, à l'époque de l'échafaud, de la hache et de la guillotine, la question du cou qu'on coupe. Du cou coupé, la coulpe n'est jamais très loin. (07.02.2001)

Autour du cou, tout cela, je l'ai autour du cou, en venant sur l'échafaudage de la scène du colloque. Parler du séminaire de Jacques Derrida, parce que je le prépare, avec Marc Crépon et Geoffrey Bennington, pour publication, c'est parler d'un texte inédit qu'on peut théoriquement aller lire en manuscrit à l'Institut des Manuscrits des Écrivains Contemporains (IMEC), mais soyons francs, je me mets en position de privilégié, en position de Vor-recht (pour parler comme Walter Benjamin2), c'est-à-dire en position de violence, et le risque est que j'essaie de tirer un profit professionnel par le biais de mon utilisation intéressée de ce privilège.

Il y a là un noeud de serpents sauvages : confession, aveu, scénarios d'Oedipe, peine et punition, culpabilité qui précède le méfait, méfait qui succède à la punition.

\footnotetext{
2 Walter Benjamin, Critique de de la violence, tr. fr. Maurice de Gandillac, revue par Rainer Rochlitz, dans Oeuvres I, Paris, Gallimard, 2000, Zur Kritik der Gewalt, und andere Aufsätze, Frankfurt am Main, Suhrkamp, 1990.
} 
Le séminaire sur la peine de mort de Jacques Derrida, par le biais de la place qu'y occupe l'argument kantien en faveur de la peine de mort, me servirait de point de départ pour lire un cas célèbre de la peine de mort, l'exécution du meurtrier et du grand criminel, Gary Gilmore, aux États-Unis en 1977. L'histoire de Gilmore est racontée dans deux livres, The Executioner's Song de Norman Mailer (1980) et Shot in the Heart de Mikal Gilmore (le frère de Gary), publié en 1995. ${ }^{3}$ Dans ce scénario, s'articuleront les idées suivantes. D'une part, le criminel qui commet un crime parce qu'il se sent impulsé par un sentiment originaire de culpabilité. Au lieu d'être la cause de la culpabilité, le crime est l'effet d'une culpabilité qui précède le crime en en étant la cause. D'autre part, la possibilité que le criminel vient à occuper la place du juge, que les positions du criminel et de la loi se verront être renversées.

En essayant de lire Kant avec les yeux de Gary Gilmore, mon pari est que cela donnerait la position de Derrida. Le recours à Gilmore, c'est-à-dire à l'écrivain Norman Mailer, afin de lire Kant répète l'avis méthodologique de Derrida qui, lui, recourt à Kafka pour déplier l'argument kantien. Plus que du côté de la science politique, de la sociologie, de la philosophie ou d'autres de tels discours, c'est du côté de la littérature que Derrida trouve le levier le plus efficace pour expliquer le texte philosophique kantien.

Je veux maintenant articuler, pour ainsi dire, le cas du criminel Gary Gilmore qui veut être exécuté par l'État d'Utah, avec le cas du criminel selon Kant qui dans un sens très particulier veut être légalement exécuté. Le mot avec lequel ces deux cas s'articulent est l'allemand Befugnis ou befugen, utilisé trois fois dans la section de Kant consacrée à la peine de mort. La discussion de Derrida, dans Spectres de Marx du mot fugen 4 , qu' on peut traduire par « joindre » ou par, en anglais, le verbe rare de « to fay [joindre] », est en arrière-plan, mais Derrida ne relève pas ce mot dans son séminaire.

Premier exemple : Kant imagine le cas d'un meurtrier condamné à mort qui se plaindrait que la peine de mort était excessive et donc injuste : tout le monde lui rirait au nez. Si tout le monde ne riait pas, si on le prenait au sérieux, dans ce cas où selon la loi ce n'est que le droit qui s'applique au criminel, alors nous devrions tous conclure que « la violence législative de l'État n'est pas apte (nicht befugt, n'est pas compétente ou légitime, n'a pas l'autorité) à prononcer ou imposer cette sorte de peine, et si [la violence législative de l'État] le fait, elle sera en contradiction avec elle-même $»^{5}$. Nicht befugt ici signifie que la violence légale de l'État serait inéga-

3 Le livre de Mailer fut publié en 1979, et est disponible avec Vintage International, traduit en français sous le titre de Le Chant du bourreau, tr.fr., Jean Rosenthal, Paris, Robert Laffont, 1980, 2008. Les numéros de page renvoient à l'édition américaine (ES) et à l'édition française (CB), traductions modifiées. Shot in the Heart. One Family's History in Murder, de Mikal Gilmore, London, Penguin Books, 1994, désormais abrégé SH en parenthèses.

4 J. Derrida, Spectres de Marx, Paris, Galilée, 1993, pp. 42-59.

5 I. Kant, « Vom Straf und Begnadigungsrecht » (Du droit de punir et de gracier), Die Metaphysik der Sitten, p. 334 (édition de l'Akademie), my translation. Désormais cité : Kant, p. X entre parenthèses. 
le à elle-même si les deux conditions obtenaient : le condamné se plaint, et le peuple ne lui rit pas au nez. Je soulève cet exemple parce que dans le cas de Gary Gilmore non seulement il ne se plaint pas, il oblige l'État à le tuer. Gary Gilmore, dont le nom légal était Fay Coffman, maintient la loi ensemble avec elle-même, forme la suture entre le punisseur et le puni.

C'est comme si Kant avait prévu l'idée troublante qu'un criminel pourrait luimême être ce qui permet à la loi d'être égale à elle-même, pleinement justifiée. Car immédiatement Kant essaie d'invalider l'idée que quelqu'un pourrait vouloir être puni. Le contexte est son désaccord avec l'argument de Cesare Beccaria contre la peine de mort. Selon Beccaria, la peine de mort et le contrat social sont incompatibles parce que la promesse, nécessaire à chaque individu, d'accepter de mourir s'il commet un meurtre, voudrait dire que chacun veuille, dans ce cas, disposer de sa vie, ce qui est cependant impossible. Kant tient cela pour du sophisme et de la perversion du droit. Pour Kant, personne ne se soumet à une punition parce qu'il la voulait, mais parce qu'il a voulu faire un acte punissable. Kant pense que le problème est ainsi évité, vu son enchaînement rhétorico-pseudo-logique: « car ce n'est pas une punition, quand ce qui arrive à quelqu'un est ce qu'il voulait, et c'est impossible de vouloir être puni ». Dans son séminaire, Derrida tournera cette affirmation de tous les côtés, et c'est le lieu où Derrida déconstruit Kant. L'argument de Kant est que c'est l'homme rationnel en moi qui soumet, qui sujette, l'homme sensible en moi, aux lois punitives. L'homme rationnel met l'homme sensible à la disposition de l'homme rationnel. Tout se passe, tout passe, à travers cette distinction, cette limite entre les deux personnes, entre ces deux « autres », l' " autre personne » en tant que « homme rationnel » qui est « un autre que » l'autre autre, à savoir « le criminel ». Derrida en dira pourtant : si une telle rationalité existait, le coupable doit « approuver et exiger sa condamnation à mort » et symboliquement au moins «participer à son exécution » qui est la « vérité d'un suicide» (31.1.2001). En jeu ici, le danger ici, est la confusion entre exécution et suicide, hétéro-punition et auto-punition, loi et criminel, et entre punition, crime et culpabilité. L'homme rationnel et l'homme sensible risquent d'être confondus, l'un et l'autre, l'un pour l'autre. L'un risque de se faire prendre pour l'autre, dépend de l'autre, de se faire pendre pour l'autre.

C'est à ce lieu que Kant réfute l'idée selon laquelle on peut vouloir se laisser punir, ayant recours à la racine de fug. "Car, si une promesse de la part du malfaiteur de vouloir se laisser punir devait se loger à la base de l'aptitude de punir (Befugnis zu strafen, autorité, capacité, légitimité à punir), alors il faudrait aussi lui abandonner la tâche de se trouver passible de peine, et le criminel serait son propre juge » (Kant p. 335). En d'autres termes, si le criminel est capable de maintenir une promesse envers lui-même selon laquelle lui en tant que criminel accepterait d'être considéré comme susceptible, passible de peine, straffällig, alors cela signifierait 
que toute distinction entre criminel et juge tomberait, s'effondrerait. Straffällig, traduit par " passible de peine », est composé de «fällig », de Fall, cas, " promis à tomber », peut-on dire peut-être. Le sens de fällig est échoir. Le danger, pour Kant et que veut écarter Kant, est que l'autorité à punir (Befugnis zu strafen) pourrait se confondre, être confondue avec la volonté de se laisser punir, voire avec le zèle de se trouver punissable, coupable. Le danger est que le juge et le criminel reviendraient au même, que le juge serait égal (gleich) au criminel, une auto-affirmation qui s'annulerait simultanément en auto-destruction.

Dans le cas de Gary Gilmore, les deux motifs qui fonctionnent comme une lecture derridienne de Kant, concernent d'une part le désir ou la soif de mort et de punition, notamment pour apaiser une culpabilité qui appartient à son père, et, d'autre part, le devenir-juge du criminel. Commenter le cas de Gilmore, c'est un peu lire Kant avec les yeux de Derrida ${ }^{6}$. Mon but n'est pas de défendre Gilmore contre la peine de mort, mais de le lire par rapport à Kant.

Premièrement, vouloir la mort, vouloir la punition.

Gilmore fait un rêve récurrent dans lequel il est décapité (ES 137), un autre dans lequel il est mis dans une boîte qui est insérée dans un trou d'un mur qui se ferme par une porte de four. Le rêve de décapitation, il l'associe à son désir de payer ses dettes, mais il fait ce rêve depuis l'âge de neuf ans. Dans leur maison dont toute la famille, même le père sceptique, est persuadée qu'elle est hantée, l'enfant de neuf ans crie comme s'il était étranglé, et sa mère entre pour voir un incube assis sur sa gorge et qui disparaît quand elle entre (ES 312). À cette date, ses rêves d'être exécuté commencent et ils ne le quitteront jamais (même dans le couloir de la mort, ES 362). Une voisine, juive, les avertit que leur maison est hantée. À la même époque, Gary décide qu'il veut être prêtre. Déjà quand il n'avait que trois ans, sa mère savait qu'il allait être exécuté, et elle vivait avec cette peur depuis lors. Bien avant son procès, quand le premier policier l'interroge, officieusement, et que Gilmore ne sait même pas combien d'argent il a volé, le policier lui dit qu'il ne comprend pas qu'on puisse prendre la vie à quelqu'un pour si peu d'argent. Gilmore répond qu'il ne sait pas ce qu'il a pris, et il demande le montant au policier. Quand il l'apprend, il dit tout de suite, « J'espère qu'ils m'exécuteront pour cela. Je dois mourir pour ce que

\footnotetext{
6 En 1979, le groupe punk The Adverts écrit la chanson, "Looking Through Gary Gilmore's Eyes » (www.youtube.com/watch? $v=$ CrwI1gKE4jI). Gary Gilmore a fait don de ses organes, et de ses yeux à une personne qui lui avait écrit pour les lui demander après l'exécution. Gary Gilmore, qui voulait mourir au lieu de rester en prison, avait tenté deux fois de se suicider. Mais en cas de mort par suicide, le don d'organes n'est pas autorisé. Parmi ses autres raisons, Gilmore voulait être exécuté par l'État aussi parce que c'était le seul moyen pour faire don de ses organes (outre, bien sûr, une mort naturelle, mais Gilmore, à 35 ans, était en bonne santé). The Adverts sont très attentifs au déplacement des yeux de Gilmore, qui lui survivent et continuent de voir après sa mort, d'une façon panoptique, transcendante. The Adverts nous donnent à voir comment on peut voir avec les yeux de l'autre, après sa mort.
} 
j'ai fait » (ES 296). À ses premiers avocats, qui veulent faire appel de la condamnation à mort, il proteste : " voyons, est-ce que je n'ai pas le droit de mourir? (Il les dévisagea.) Est-ce je ne peux pas accepter ma peine ? „Et il ajoute qu'il croit qu'il a déjà été exécuté une fois, au dix-huitième siècle en Angleterre. "Je sens que j'ai été ici déjà. Il y a quelque crime de mon passé .... Je sens qu'il faut que j'expie la chose que j'avais faite alors» (ES 490).

La jeunesse de Gilmore est marquée par les coups d'une extrême violence qu'il reçoit de la main et de la ceinture de son père toutes les semaines jusqu'à l'âge de 14 ans, quand il est mis en prison, où il restera pour les vingt deux ans qui lui restent à vivre, à part quelques mois de liberté. Avant son adolescence enfermée, Gilmore aimait courir sur une voie ferrée suspendue dès qu'il voyait un train émerger d'un tunnel et venant vers lui. Le jeu consistait à courir la distance du pont et quitter le pont avant que le train, à seulement quelques mètres de lui, ne l'attrape et l'écrase. Bien plus tard, dans le couloir de la mort, il est interviewé (entretien qui est vendu à Playboy Magazine pour 25 mille dollars); l'intervieweur lui dit: « en lisant vos dossiers de prison, il nous semble que vous avez été bouclé presque continuellement depuis que vous avez été mis en école de redressement il y a vingt-deux ans. C'est comme si vous n'aviez jamais eu d'autre choix que de vivre (live out) un destin criminel » (ES 794, CB 974). À quoi Gilmore dit, " c'est très bien dit ». Après son arrestation pour les meurtres, Gilmore est bien sûr examiné pour déterminer s'il est malade mental. Bien que considéré comme " psychopathe » ou comme ayant de une personnalité « psycho- » ou « sociopathe », il n'est pas diagnostiqué comme "psychotique ». Son médécin explique aux avocats de Gilmore que la loi veut garder «psychopathie » bien séparée de «psychose » : psychose c'est l'hôpital ; psychopathie c'est la prison. Si le psychopathe devait jamais être reconnu légalement malade mentalement, leur dit-il, alors le crime, le jugement et la punition seraient remplacés par l'acte antisocial, la thérapie, et la convalescence (ES 385). Ici s'insérerait la lecture des écrits de Theodore Reik et de Sigmund Freud que Derrida développe sur plusieurs séances de la deuxième année du séminaire, en particulier par rapport à la peine de mort, à la punition, au crime et à la culpabilité. La culpabilité et même l'intuition inconsciente d'une culpabilité précèdent le crime ; la culpabilité n'est pas l'effet du crime, elle en est la cause. On tue parce qu'on se sent coupable, ceci valant aussi bien pour les criminels que pour l'appareil de l'État qui exécute la peine de mort. La psychanalyse permettra(it) à déjouer cette causalité obscure du meurtre par le criminel et par l'État. La psychanalyse remplacerait la loi, la thérapie, le châtiment, et la poena naturalis ou l'auto-analyse remplacerait la poena federalis ou la hétéro-punition. (07.02.2001). Poursuivons avec Gilmore. Freud et Reik se réfèrent à un texte de Nietzsche que Derrida ne soulève pas, je mentionne ici le passage extraordinaire sur « le pâle criminel » (Von dem bleichen Verbrecher) dans Ainsi parlait Zarathoustra, parce que dans ce texte de 
Nietzsche le criminel cherche à être puni pour une culpabilité sans cause, sans crime, mais aussi parce que Gary mentionne Nietzsche à son frère Mikal venu lui rendre visite dans le couloir de la mort quelques $72 \mathrm{~h}$ avant son exécution. Mikal, dans une lettre transmise à Gary pour qu'il puisse la lire pendant sa visite, essaie d'expliquer à son frère les raisons pour lesquelles sa mère et lui pourraient tenter de bloquer l'exécution contre la volonté de Gary. Après l'avoir lue, Gary lui répond lors de sa visite: «c'est bien écrit : est-ce que tu as lu Nietzsche ?» (SH 484). Ici un extrait de Zarathoustra sur le pâle criminel qui décrit bien Gilmore :

Écoutez, juges ! Il y a encore une autre folie : et cette folie est avant l'acte. Hélas ! vous n'avez pas pénétré assez profondément dans cette âme !

Ainsi parle le juge rouge : "Pourquoi ce criminel a-t-il tué ? Il voulait dérober." Mais je vous dis : son âme voulait du sang, et ne désirait point le vol : il avait soif du bonheur du couteau!

Mais sa pauvre raison ne comprit point cette folie et c'est elle qui décida le criminel. "Qu'importe le sang! dit-elle ; ne veux-tu pas profiter de ton crime pour voler? pour te venger?"

Et il écouta sa pauvre raison : son discours pesait sur lui comme du plomb, - alors il vola, après avoir assassiné. Il ne voulait pas avoir honte de sa folie. ${ }^{7}$

Gilmore est jugé dans l'État d'Utah, connu pour son histoire et ses institutions fondées sur 1' « expiation par le sang », «blood atonement » (SH 3-30). De prison, il écrit à son amante : «bientôt je serai plus pâle qu'un fantôme. En fait, bientôt, je pourrais bien être un fantôme » (ES 330 ; CB 412). En prison, il plaisante avec son compagnon de cellule concernant ce qu'il a dit à l'employé de l'hôtel avant de le tuer: « Ton argent, fils, ET ta vie » (ES 357 ; CB 446) ; mais dans tous les récits concernant ce qui s'est passé, Gilmore ne se souvient pas de l'argent ou d'en avoir pris (ES 441, 296, 735). Il se rappelle son meurtre mais non pas son vol d'argent. L'obsession qu'a Gilmore - son obsession de sa future exécution inéluctable qui l'accompagne pendant des décennies - tient à son sens aigu des crimes de son père. Gilmore est au courant des crimes de son père, d'abord de manière plutôt obscure pendant son enfance, et puis, à l'âge adulte, de manière spécifique mais secrète (secret qu'il partage avec sa mère et ses frères n'ont jamais pu percer). En Gilmore, il y a un cas d'école de ce que décrivent Reik et Freud : le criminel qui commet un crime pour avoir une cause de la culpabilité archaïque qu'il ressent et qui cherche à être pris pour que sa culpabilité puisse être punie.

Après les deux meurtres, pendant et après son procès, Gilmore ne ressent rien pour ses victimes. Le fait de la poena federalis qu'il doit subir ne luit fait aucun effet ; qui plus est, il dit dès ses aveux officieux et surprenants (c'est un criminel

7 Friedrich Nietzsche, Ainsi parlait Zarathoustra. http://fr.wikisource.org/wiki/Ainsi_parlait_ Zarathoustra/Premi\%C3\%A8re_partie/Du_p\%C3\%A2le_criminel 
très dur qui de sa vie n'a jamais rien avoué à un policier) qu'il doit être exécuté (ought to be executed), et ce n'est que quand il apprend qu'il avait volé un peu d'argent que quelques larmes lui échappent. Mais, en prison, il apprend qu'un ami de sa première victime a tenté d'entrer dans la prison, en se faisant passer pour un avocat, afin de pouvoir venger son ami en tuant Gilmore. C'est en apprenant cette nouvelle que Gilmore sent de la compassion et dit avoir pour la première fois du sentiment pour les deux gars qu'il a tués : Que vaut un mort, s'il n'avait pas d'amis pour le venger? (ES 387). L'ami voulait effectuer ce que Kant appelle la poena naturalis, un acte de vengeance. Pour Gilmore, sa victime n'a de valeur qu'à partir d'une logique de la poena naturalis. À l'inverse, la Cour Suprême de l'État d'Utah, dont on s'attendrait qu'elle ne prenne appui que sur une poena federalis, décide, à 07:35, d'annuler l'ordonnance de surseoir qu'un juge avait prononcé à 01:05 du matin même de l'exécution (qui était programmée au lever du jour, à 07:42). L'ordonnance de surseoir par le juge Ritter se basait sur le fait qu'il n'avait pas été prouvé qu'il était constitutionnel dans l'État d'Utah de ne pas avoir un appel automatique en cas de peine de mort. Les juges de la Cour suprême de l'État ont été réunis en urgence. Avant de livrer leur verdict, un juge parle pour dire que « $\mathrm{M}$. Gilmore a ses droits. Si une erreur est en train de se commettre dans la poursuite de cette exécution, c'est lui qui l'a provoquée » (ES 965 ; CB 1186). Avis extraordinaire. Les juges préfèrent prendre le risque qu'il y a une erreur dans la constitution que le vérifier en maintenant l'ordonnance de surseoir, car ils considèrent que s'il y a une lacune dans la poena federalis - absence d'un appel automatique - c'est le condamné qui en est de facto responsable. L'individu, le condamné, qui opère à un niveau de poena naturalis (il veut être puni), permet aux juges de ne pas examiner l'éventualité qu'une erreur judiciaire est en train de se faire.

L'ambiguïté entre la poena naturalis (une peine subjective) et la poena federalis (la peine objective, juridique) est claire quand, quelques jours avant la date de l'exécution, Gilmore écrit une lettre publique à sa mère pour lui demander de ne pas déposer une demande d'appel à sa place. Gilmore dicte sa lettre à ses avocats (qu'il paie pour ne pas le défendre contre la peine de mort, ils doivent accepter cette condition avant qu'il ne les embauche): « chère maman. Je t'aime profondément, je t'ai toujours aimée, et je t'aimerai toujours. (Pause) Mais s'il te plait dissocie-toi de cet oncle Tom, la NAACP. S'il te plaît, accepte le fait que je souhaite être mort (wish to be dead). Que je l'accepte. Que je l'accepte ». Son avocat Moody: « Voulez-vous mettre "que je l'accepte" plus d'une fois? » Gilmore: « S'il te plait, accepte le fait que je veux, que j'accepte, la mort. Comment le dire mieux que ça? S'il te plaît, accepte cela ». Avocat Moody : « Peut-être, s'il te plaît, accepte le fait que j'accepte ce qui m'a été imposé par la loi, c'est cela que vous essayez de dire ? » Gilmore: «Ouais. Cela irait. Je ne veux pas que cela ressemble à un voeu de mort (a death wish ; Rosenthal traduit par « un instinct de mort ») en disant que je sou- 
haite la mort (I wish for death)». Moody : « J'accepte simplement ce qu'est la loi ». Stanger (l'autre avocat) : «Appliquer la loi ». Gilmore : «Heu, j'aimerais te parler. J'aimerais te voir. Mais je ne peux pas, donc je t'envoie cette lettre par l'intermédiaire du journal. (Longue pause.) On meurt tous, il n'y a pas de quoi en faire un plat» (ES 696 ; CB 857). Difficile de voir l'homo noumenon de Kant dans les dires de Gilmore, mais facile de le voir dans les dires de ses avocats. Quand les médias couvrent Gilmore, et la manière dont il oeuvre pour accélérer sa propre exécution, ils parlent de son plea for death, son " appel » ou « argument » pour et en faveur de la mort, comme si le fait qu'il a tout fait pour l'avoir revenait à un argument au sens juridique. Et Gilmore console son deuxième avocat (le premier à avoir été embauché sous condition qu'il l'aide à être exécuté selon la lettre de sa condamnation) lorsque celui-ci lui dit qu'il a le sentiment d'être Judas en l'aidant à être exécuté. Judas, lui dit Gilmore, était l'homme le plus injustement critiqué, le plus calomnié (most bum-beefed) de l'histoire (ES 527). Judas savait ce qui allait se passer. Judas était là pour aider Jésus à être branché sur, à se faire à, la prophétie (ES 527, tune in). Sans faire de Gilmore une figure du Christ (il s'identifie plutôt au diable), il y a néanmoins ce point commun qui consiste dans le fait que, si Jésus n'a pas commis de crime, mais avait besoin d'une punition, d'une peine de mort pour répondre de et à une culpablité originaire, Gilmore agit comme quelqu'un devant expier des crimes ancestraux ou archaïques où le crime de meurtre semble n'avoir d'autre raison que d'appeler la punition de ce sentiment de culpabilité originaire.

Deuxièmement, pour compliquer d'avantage le schéma kantien, ou pour lire en Gilmore l'implicite que reconnaît très subtilement Kant, il y a un devenir-juge du criminel dans l'histoire de Gilmore, une transformation de l'hétéro-punition (qu'il doit subir) en auto-punition (qu'il réclame), et par effet inverse, de l'auto-punition (que l'État est censé décider et appliquer) en hétéro-punition (que l'État est contraint d'appliquer).

Dans son séminaire, Derrida insiste constamment sur le rôle des médias dans la création du spectacle, dans la spectacularisation de la peine de mort. Cet aspect, très important dans le livre que Norman Mailer consacre aux exécutions de Gary Gilmore, passe ici sous un quasi-silence. Gilmore est à la une des journaux nationaux, régionaux et locaux. Il figure à la une des hebdomadaires les plus répandus (Time, Newsweek). Des films de télévision et de cinéma mettant sa vie en scène sont réalisés (exemplifiant ce que Walter Benjamin, dans Critique de la violence, appelle l'admiration secrète pour le criminel). On peut noter pourtant que le livre comporte de nombreux extraits des journaux de l'époque. Lorsque la Cour Suprême des États Unis « ruled that Gary Mark Gilmore had made a knowing and intelligent waiver of his rights » (la Cour suprême des États-Unis a décrété que Gary Mark Gilmore avait renoncé en toute connaissance de cause à faire valoir ses droits), le titre de l'article de presse, ce qu'on voit en premier, est « No More Delays Gilmore 
Says » (ES 733 ; «Plus de retards, dit Gilmore »). Bien que ce soit la Cour qui statue, le journal donne à penser que c'est Gilmore qui est le décideur. Pendant toute la période entre la condamnation à mort et l'exécution, il y a une bataille entre la Cour et Gilmore pour déterminer qui est dans la position juridique décisive. Si la Cour lui rappelle que ce n'est pas lui qui s'est condamné à mort, et que ce n'est pas lui qui décide, il reste vrai que la Cour fait presque toujours ce qu'il veut et ce qu'il la défie de faire. Quand son droit de ne pas faire appel semble accepté par la Cour Suprême américaine, la Cour d'Utah invoque la prescription de 30 à 60 jours entre la décision et l'exécution. Le procureur suggère une date, 30 jours plus tard, pour l'exécution. Quand c'est au tour de Gilmore de parler, il explose devant la cour, les accusant de ne pas avoir assez de tripes ou de couilles pour le laisser mourir, il les accuse de jack him around (manipulé, avec un sens de masturbation), et les défie : si tout cela n'est pas une blague, il s'attend à ce que sa sentence soit appliquée dans les jours à venir. Le moins que la justice puisse faire c'est de le reconnaître. Le juge fixe la date dans 31 jours, disant à Gilmore que la Cour n'est pas là pour « se plier à vos voeux ». La Cour refuse, mais la Cour est constamment défiée, directement ou indirectement dans les journaux, par Gilmore. Et la Cour donne la date la plus rapide possible. Gilmore lui-même veut l'exécution la plus rapide possible selon une logique de la poena naturalis. Et quand il est arrêté après les meurtres, il se met en colère contre sa cousine qui avait dit à la police où il se trouvait. Elle le console en disant qu'au moins il est vivant, à quoi il répond qu'il aurait été beaucoup plus simple si la police l'avait zigouillé, buté, sur la Highway 89, route du farwest. Une telle poena naturalis aurait été plus simple et surtout très rapide, mais toute la confrontation entre Gilmore et l'État d'Utah sera une bataille, pour l'un et l'autre, pour obtenir la seule peine légale tout en la rapprochant le plus possible d' une peine naturelle. Aussi, Gilmore est condamné à mort, il reçoit une hétéro-punition, mais ce sera lui qui obligera l'État à l'appliquer contre le gré de l'État, suite à quoi la peine ressemble à bien des égards à une auto-punition de Gilmore par Gilmore, et dont l'État est plus ou moins obligé de reconnaître qu'elle devient, en ce qui le concerne, une hétéro-punition, une peine commandée par le condamné et infligée à l'État.

Lorsque la condamnation à mort est prononcée, le juge demande à Gilmore quelle forme d'exécution il veut. Gilmore choisit d'être fusillé. Il y aurait beaucoup à dire sur ce choix, étant donné l'histoire des exécutions et l'histoire de l'expiation par le sang (blood atonement) dans l'État Mormon d'Utah. Notons plutôt que le procureur en ce moment même regarde toutes les personnes dans la Cour et considère que la seule personne qui n'est pas folle est Gilmore, et que Gilmore est la plus intelligente et la mieux éduquée (bien qu'il ait quitté l'école à 15 ans). Souvent, il y a de la confusion quand il faut se demander qui est le plus souverain, le juge ou le condamné. Dans la presse écrite du 2 novembre, le journal local titre que Carter 
gagne l'Élection présidentielle (nous sommes en 1976), que le juge ordonne un examen du condamné, et l'article précise que si Gilmore gets his way, obtient ce qu'il veut, il sera la première personne exécutée dans l'Utah depuis 16 ans (ES 511). La chaîne de la hiérarchie (Président, juge, condamné) semble être respectée, mais la presse parle de ce que veut Gilmore, comme si la poena federalis, et l'hétéro-punition, dépendaient en quelque sorte de son désir subjectif, d'une forme d'auto-punition. Et, suite à un appel téléphonique qu'il fait à l'Assistant Attorney General (qui est du côté du procureur), Gilmore obtiendra un avocat favorable à la peine de mort et qui promet de ne rien faire pour bloquer son application (ES 518-19). L'écrivain qui conduit l'entretien pour Playboy considère que Gilmore exige de toutes les parties un comportement civilisé exquis dont presque aucun représentant de la loi n'est capable (ES 647). Les distinctions que Kant veut tenir entre l'homo noumenon qui accepte la loi et l'homo phaenomenon qui veut être puni sont bien brouillées quand Gilmore pousse tant d'hommes de loi à reconnaître, explicitement ou implicitement, son avantage.

Gilmore lance un défi à la loi, dès son incarcération avant son procès. Il dit à son camarade de cellule: "ils comptent m'infliger la peine de mort. Mais j'ai une réponse à leur faire. Je vais jouer la carte cachée de l'État d'Utah (I'm going to check into the State of Utah's hole card. Je vais les forcer (make them) à le faire. Nous allons alors voir s'ils ont autant de tripes (as many guts, autant de cran) que moi" (ES 358 ; CB 447 ; la métaphore du jeu de cartes file à travers la deuxième année du séminaire de Derrida sur la peine de mort). On reconnaît là ses défis aux trains lorsqu'il n'avait que 12 ou 13 ans. Sa seule crainte est qu'un ami de la victime sera parmi les tireurs : si cela devait être le cas, le tireur viserait sa tête, ce que Gilmore ne veut pas, car ses yeux parfaits, il veut en faire don. Renversant la situation de telle sorte que l'État est dépossédé de sa position de maître du jeu, Gilmore craint néanmoins un ultime renversement de son renversement selon lequel la poena federalis redeviendrait une poena naturalis, lui serait volée au dernier instant.

Lorsque ses premiers avocats, déjà virés par lui, obtiennent un report de l'exécution, Gilmore écrit le jour même à la Cour: « est-ce que le peuple d'Utah n'a pas le courage de ses convictions? Vous condamnez un homme à mort - moi - et quand $\mathrm{j}$ 'accepte cette punition la plus extrême (this most extreme punishment ; Rosenthal traduit "ce châtiment suprême") avec grâce et dignité, vous, le peuple d'Utah, voulez céder et en discuter avec moi. Vous n'êtes pas sérieux » (ES 521 ; CB 642). L'ambiguïté concernant qui commande est lisible dans les journaux. La décision de la Cour Suprême d'Utah de permettre (allow) à Gary Mark Gilmore de mourir devant un peloton d'exécution est annoncée à la première page des plus grands journaux de New York et de Washington. Mais l'annonce de cette décision dit que la Cour va allow, permettre, à Gilmore de mourir de cette façon, comme si 
la Cour confère un statut légal à une volonté naturelle du condamné. Bien que dite par la presse, l'annonce donne l'impression que la loi n'est là que pour cautionner et permettre un dispositif technique, aux frais de l'État, pour satisfaire au désir du condamné. Il critiquera, dans les journaux, le gouverneur pour avoir différé la date de l'exécution, en défendant son propre " "welfare" ", son " "bien-être" ", consistant en sa mort (ES 548 ; CB 676) ; après sa tentative de suicide, parce qu'on lui obtient des sursis qu'il ne veut pas, ou parce que l'exécution doit attendre une trentaine de jours, son chaplain, son conseiller spirituel (Campbell) l'encourage en lui disant que Gilmore peut «mettre la loi à l'épreuve ». S'il se suicide, rien n'est résolu. Il l'exhorte à « les forcer à aller jusqu'au bout» (force them to the issue, ES 621 ; CB 764). Même si Gilmore lui rétorque que « la loi ne me signifie rien », Gilmore suit la recommandation. Le jeu consiste à obliger l'autre à réagir en premier afin d'avoir le sentiment qu'on l'a poussé à un acte dont on reste le décideur.

En face de la résolution de Gilmore, la loi, incarnée par les procureurs et les juges, se distingue par son émotivité, par son horreur de la violence. Le procureur qui a obtenu la condamnation à mort dit qu'on ne pouvait pas l'emmener de force à l'exécution, et qu'il a fait son boulot, qu'il a demandé et obtenu la peine de mort, et qu'il y croit. Mais une exécution est sale, salissante et embrouillée et il ne veut pas en faire partie (ES 561). De jure pour la peine de mort, le procureur est bien de facto contre, et en cela il retrouve parfaitement la lecture rhétorique à laquelle le texte de Kant se prête. Oui, la peine de mort, mais sans la moindre Misshandlung ! L'État est bien obligé de tuer afin d'atténuer la compulsion de punir et de tuer. Gilmore ne demande qu'à être tué, selon la loi ou selon la loi de blood atonement qui est un vestige des origines Mormones de l'État d'Utah, mais, comme le prévoit l'écrivain pour Playboy (qui s'appelle Barry Farrell), si l'État ne le tuait pas, lui qui voulait être fusillé, alors un tsunami d'exécutions s'ensuivrait. Chaque conservateur demandera : qui allons-nous jamais punir, châtier, après cela ? (Who are we ever going to punish? ES 639 ; CB 787).

Gilmore ne cesse de railler l'État à propos de son hésitation à l'exécuter. Chaque fois qu'une instance doit se réunir pour décider d'un sursis possible ou d'un appel, Gilmore publie un article dans la presse la veille qui les accuse de couardise. Dans une lettre publiée dans le journal et adressée au Conseil des Pardons, Gilmore leur écrit, " alors, faisons-le, vous les lâches, les poltrons », précisant qu'il demande une exécution immédiate, et qu'il ne cherche ni désire leur grâce, soulignant « ne » trois fois (ES 672 ; CB 826). À railler autant, à avoir recours aux médias, à attaquer juges, procureurs, avocats et hautes instances, Gilmore a une influence qui est certes impossible à mesurer, mais qui sans doute rend douteuses les dénégations faites par lesdites instances lorsqu'elles proclament que ce sont elles qui décident et Gilmore qui subit.

Craignant que les tentatives de suicides obligent l'État à renouveler l'examen de 
l'état de santé mentale de Gilmore, l'Assistant Attorney General argue que les suicides plaident en faveur du maintien de l'exécution. Laisser Gilmore en prison avec une longue peine serait pire que le tuer rapidement. Pour lui, une exécution est comme un big bonfire qui permet de maximiser l'essence de l'étincelle de l'humanité (décision de tuer) peut même augmenter son essence (matière flammable) en choisissant une alternative (peine de mort) qui sauvegarde la plus grande dignité (la mort par châtiment, et non pas la continuation d'une vie exécrable) (ES 706 ; CB 869). Si Gilmore inspire l'argumentaire de cet Assistant Attorney General, il inspire non pas l'admiration mais plutôt le respect du Assistant District Attorney, Earl Dorius, parce qu'il trouve chez Gilmore que la loi, la violence de la loi, est respectée. Si Dorius écarte le mot d' " admiration » (l'admiration secrète du peuple pour le grand criminel, supposée par Walter Benjamin), il reste que Gilmore suscite le respect de Dorius parce que Gilmore présente un modèle à suivre au District Attorney quand il s'agit de respecter la loi. En acceptant pleinement son exécution et en l'accélérant, Gilmore gagne sa propre rédemption (ES 679). Dorius est fier de Gilmore (took pride in Gilmore) car le condamné respectait la situation. Il serait désagréable pour Dorius de travailler sur un dossier d'une importance aussi capitale s'il sentait que l'individu à son centre n'était qu'une petite canaille au mobile douteux (shoddy motives). Et Dorius, qui trouve le désir de Gilmore genuine (authentique), en fait un role model pour son propre travail en tant que District Attorney qui doit garantir l'avoir-lieu de l'exécution. Véritable miroir d'amour spéculaire entre le condamné et le condamnant-bourreau.

Presque en coda ici, et glissé subrepticement dans l'Executioner's Song de Mailer est une trouvaille d'importance majeure (que Mikal Gilmore élucide davantage aussi) et qui embrouille totalement les oppositions entre hétéro-punition et auto-punition et entre disons la philosophie de la peine de mort (à la Kant) et la psychanalyse de thérapie (à la Reik) et dont Derrida développe mieux que nous quelques contours. C'est une page trop riche pour être suffisamment commentée ici. Qu'il suffise ici de noter que Gilmore reconnaît une pulsion pour les adolescents, garçons ou filles, et que ses meurtres étaient le moyen de s'empêcher à agir sur ses pulsions tout en se punissant pour le fait d'avoir eu ces pulsions. Les meurtres suivaient directement la séparation avec son amante, Nicole, qu'il appelait sa fée, et qu'il comparait à une elfe et à un être adolescent au tout début de la maturation sexuelle. L'écrivain de Playboy qui découvre cette possibilité la relie au sens de noblesse que Gilmore dérivait de ses meurtres, comme si les meurtres étaient une forme de punition, certes, mais aussi une forme d'honneur dans la mesure où il s'est empêché de commettre des actes de loin plus ignobles. Il tue les autres afin de tuer, en lui, cette personne dont il ne pourrait qu'avoir honte. Les meurtres lui permettent, certes de manière pervertie, de garder un certain sens de l'honneur (ES 882). À cette hypothèse, on peut associer l'idée farfelue de Gilmore, quand il apprend 
seulement six heures avant son exécution qu'un juge venait de prononcer un sursis parce qu'il était possible que l'argent du contribuable allait être dépensé de manière illégale, l'idée farfelue de Gilmore de payer lui-même de sa poche le montant de l'exécution. À presque deux heures du matin, alors que l'exécution est programmée pour 07:42, Gilmore tente de tout mettre en oeuvre pour contacter l'Attorney General de l'État (le plus haut procureur) pour lui dire qu'il payerait les balles, les fusils, et les tireurs. À partir du moment où les meurtres étaient des auto-punitions destinées à provoquer une hétéro-punition par l'État dont il restait pourtant largement maître, ne serait-ce que par le biais de la raillerie et des menaces qui ne pouvaient que trouver une certaine sympathie secrète auprès des juges favorables au blood atonement, Gilmore est prêt à financer l'exécution publique de l'État par ses propres moyens privés, gagnés par la vente de ses droits dans les films et entretiens et livres qui étaient en train de se réaliser pendant ses dernières semaines de vie.

Après le dernier échec des avocats indépendants de différer l'exécution, l'avocate ultime, Judy Wolbach, compare les juges, qui ont, suivant un mobile de poena naturalis dont on a déjà parlé (exprimé par le juge Lewis), pris le risque d'une erreur à attribuer a priori à Gilmore, aux Pharisiens : toujours la lettre de la loi (ES 952). C'est une page dense que Mailer consacre à l'analyse faite par Wolbach du blood atonement, de la soi-disant nécessité de faire couler le sang afin de racheter la rédemption, qui caractérise la théocratie des seigneurs d'Utah. Mais si les Pharisiens d'Utah, comme ceux décrits par Kant, ont une arrière pensée, qui consiste à imposer la peine de mort pour une fin « utilitarianiste », une fin extrinsèque à une punition pure et simple, une punition ne relevant que d'une impossiblement simple loi du talion, le même ne peut pas être dit pour Gilmore qui se révèle plus Kantien que Kant ou aussi Kantien que Kant sans être Pharisien. Gilmore est littéraliste : il faut la mort, sa mort, pour la mort, mais ce n'est point « utilitarianiste » à la différence des juges Pharisiens déplorés par Judy Wolbach. Quoique ... ce KantGilmore sera perçu tout autrement si nous le voyons et le lisons avec les yeux de Nietzsche, de Reik, de Derrida, et même de Mikal Gilmore, voire Gary lui-même.

Le séminaire de Jacques Derrida consacré à la peine de mort déconstruit l'échafaud de la peine de mort en montrant comment la peine de mort provient d'un fantasme de souveraineté, d'un désir de contrôler le temps, de calculer le temps, remplaçant ainsi la finitude par une infinitude ; il a recourt à la psychanalyse pour repenser le rapport entre la peine de mort et la sexualité. Sans vouloir esquisser en conclusion toutes les pistes que nous n'avons pas pu suivre ici, nous terminons par une voie qu'il ouvre et que nous avons pris dans notre lecture du cas de Gary Gilmore : c'est la déconstruction de l'opposition dedans/ dehors, nature/ culture, opposition qui soutient la philosophie de la peine de mort établie par Immanuel Kant à l'intérieur de laquelle la déconstruction est toujours déjà à l'oeuvre. Donnons le dernier mot à Derrida sur ce point : 
Cette logique de l'exécution suicidaire, cette vérité suicidaire de la peine de mort, elle est, même si le condamné n'y croit pas ou ne s'y résout pas, elle est structurellement impliquée par la logique du verdict. Comme celui-ci prétend être fondé en droit, en raison, en rationalité juridique supposée universellement partagée, le coupable reconnu coupable doit donner raison à ses juges et donc à ses bourreaux, et dès lors qu'il donne raison au droit, dès lors qu'il donne raison à la raison du droit, aux juges, au bourreau, et finalement au président qui lui refuse la grâce, dès lors que la raison a raison de lui, c'est comme s'il se jugeait, se condamnait, se suicidait, comme s'il s'exécutait luimême. Il approuve la sentence, il donne raison à la sentence, et donc il se condamne luimême à mort, et pour aller au bout de cette conséquence, il exécute lui-même la sentence par laquelle il se condamne lui-même. L'exécution est sui-cide. Il n'y a, pour l'autonomie de la raison juridique que de l'auto-exécution. Je dis bien « comme s'il se suicidait ». (31.01.01) 